\title{
Inhibitor of RAGE and glucose-induced inflammation in bone marrow mesenchymal stem cells: Effect and mechanism of action
}

\author{
MENGYI JIANG，XUEMEI WANG，PIN WANG，WEI PENG，BO ZHANG and LING GUO \\ Department of Prosthodontics, Hospital of Stomatology Affiliated to Southwest Medical University, \\ Luzhou, Sichuan 646000, P.R. China
}

Received February 12, 2020; Accepted July 12, 2020

DOI: $10.3892 / \mathrm{mmr} .2020 .11422$

\begin{abstract}
The occurrence and development of hyperglycemia-induced inflammation is associated with increased expression of receptor for advanced glycation end products (RAGE) and inflammatory factors, including IL-1 $\beta$, TNF- $\alpha$ and IL-6. Previous studies have reported that the nucleotide-binding oligomerization domain-like receptor protein 3 (NLRP3) inflammasome interacts with thioredoxin-interacting protein (TXNIP) and serves a crucial role in inflammation. FPS-ZM1 has been identified as target inhibitor of RAGE and has been shown to exert an anti-inflammatory effect in vitro. However, the underlying mechanism by which FPS-ZM1 impacts high glucose (HG)-induced inflammation in bone marrow mesenchymal stem cells (BMSCs) remains unclear. The present study explored the regulatory effect of FPS-ZM1 on HG-induced inflammation in BMSCs. Furthermore, the role of the TXNIP/NLRP3 inflammasome signaling pathway in the regulatory effects of FPS-ZM1 on HG-induced inflammation was studied. Cell viability was determined using Cell Counting Kit- 8 and western blotting was used to assess the protein expression levels of RAGE. ELISA was used to determine the levels of inflammatory markers. Reverse transcription-quantitative PCR and western blotting were used to measure the mRNA and protein expression levels of TXNIP, caspase-1, thioredoxin (TRX), NLRP3 and apoptosis-related speck-like protein containing CARD (ASC). The results revealed that in BMSCs, RAGE expression was stimulated by HG, an effect which was reversed by treatment with FPS-ZM1. In addition, HG activated inflammatory factors, such as TNF- $\alpha$, IL-1 $\beta$ and IL- 6 ; however, their levels were suppressed
\end{abstract}

Correspondence to: Professor Ling Guo, Department of Prosthodontics, Hospital of Stomatology Affiliated to Southwest Medical University, 2 Jiangyang South Road, Luzhou, Sichuan 646000, P.R. China

E-mail: 372083745@qq.com

Key words: FPS-ZM1, bone marrow mesenchymal stem cells, receptor of advanced glycation end products, inflammation, thioredoxin-interacting protein/nucleotide-binding oligomerization domain-like receptor protein 3 inflammasome when cells were treated with FPS-ZM1 or the TXNIP/NLRP3 pathway inhibitor, resveratrol (Res). Furthermore, FPS-ZM1 inhibited the mRNA and protein expression levels of TXNIP, caspase-1, NLRP3 and ASC, and promoted TRX expression, which was consistent with the effects of Res. These findings indicated that FPS-ZM1 may attenuate HG-induced inflammation in BMSCs. Furthermore, the TXNIP/NLRP3 inflammasome signaling pathway mediated the molecular mechanism underlying this effect.

\section{Introduction}

Diabetes mellitus is a disease associated with numerous complications (1). Hyperglycemia affects patients with diabetes by damaging macro- and microvessels, thus resulting in retinopathy, neuropathy and nephropathy, and cardiovascular, cerebrovascular and other serious complications $(2,3)$. Although multiple mechanisms cause diabetic complications, growing evidence has suggested that immoderate aggregation of advanced glycation end products (AGEs) may be a causative factor (4). Excessive AGEs activate and interact with the receptor for AGEs (RAGE), leading to the activation of inflammatory factors $(5,6)$. FPS-ZM1 is a specific and high-affinity inhibitor of RAGE (7). Numerous studies have reported that FPS-ZM1 exerts an anti-inflammatory effect on various cells, and on human periodontal ligament fibroblasts and human gingival fibroblasts (8-10). These findings suggested a promising application of FPS-ZM1 in preventing periodontal diseases. However, further studies are required to explore its application and importance in the medical field.

It has been reported that expression of RAGE is high in gingival fibroblasts, periodontal ligament fibroblasts and periodontal ligament stem cells in a high glucose (HG) environment (11). Under HG conditions, various inflammatory factors, including but not limited to IL-1 $\beta$, high mobility group box-1, IL-6, intercellular adhesion molecule-1 and TNF- $\alpha$, can be activated through the combination of RAGE and AGEs $(12,13)$. These inflammatory cytokines have been demonstrated to cause inflammation in periodontal tissues, including the alveolar bone and surrounding soft tissues $(14,15)$. As observed previously, IL-6, IL-1 $\beta$ and TNF- $\alpha$ largely accumulate in the periodontal tissues of patients with diabetes (16). Bone marrow mesenchymal stem cells (BMSCs) are present in the alveolar bone and possess multi-directional differentiation 
potential; these cells have an essential role in bone formation (17). It has previously been reported that the release of inflammatory cytokines promotes the proliferation of BMSCs in a HG environment, thus suggesting its application in the pathological process of periodontal diseases (18). Therefore, this study aimed to assess how HG activates inflammatory cytokines in BMSCs.

Several intracellular signaling pathways involved in the activation of inflammatory cytokines are currently being studied. Notably, to the best of our knowledge, the infection-associated proinflammatory function of $\mathrm{NF}-\kappa \mathrm{B}$ is the most widely studied (19). The thioredoxin-interacting protein (TXNIP)/nucleotide-binding oligomerization domain-like receptor protein 3 (NLRP3) inflammasome pathway has been reported to be associated with diabetes-associated inflammation (20). The NLRP3 inflammasome has been well studied and is comprised of three components: NLRP3 protein, apoptosis-related speck-like protein containing CARD (ASC) and caspase-1 (21). The NLRP3 inflammasome can detect danger signals, known as danger-associated molecular patterns (22). NLRP3 agonists can activate caspase-1, which in turn releases the proinflammatory cytokines IL-18 and IL-1 $\beta(23,24)$. The role of the NLRP3 inflammasome has been demonstrated in the pathogenic process of various diseases including, but not limited to, Alzheimer's disease, osteoarthritis and type 2 diabetes (25-27).

However, few studies have described the mechanism through which the NLRP3 inflammasome is activated. Functionally, TXNIP dissociates from thioredoxin (TRX), combines directly with NLRP3 and activates it (28). In addition, recent studies have demonstrated that TXNIP is upregulated in diabetes-related inflammation $(20,29)$. In patients with diabetes, hyperglycemia has been suggested to stimulate the expression of TXNIP (30). Therefore, it may be hypothesized that HG activates the NLRP3 inflammasome by promoting TXNIP expression.

The aim of the present study was to assess the expression of RAGE in BMSCs under HG stimulation, and to investigate the expression of related inflammatory cytokines and the potential molecular mechanisms in response to a RAGE-specific inhibitor. Consequently, the feasibility of using RAGE as a target for drug therapy to prevent periodontal inflammation and promote the healing process of bone tissue injury in patients with diabetes was considered.

\section{Materials and methods}

Cell culture. BeNa Culture Collection; Beijing Beina Chunglian Biotechnology Research Institute provided the rat BMSCs. Cells were cultured under normal glucose (NG; $5 \mathrm{mM}$ ) or HG (25 mM) conditions in DMEM (HyClone; Cytiva) supplemented with 10\% FBS (Gibco; Thermo Fisher Scientific, Inc.) and $1 \%$ penicillin-streptomycin solution (Beyotime Institute of Biotechnology). Cells were cultured in a humidified atmosphere containing $5 \% \mathrm{CO}_{2}$ at $37^{\circ} \mathrm{C}$. Through the cell viability assay, an optimal concentration of FPS-ZM1 (500 nM; Beyotime Institute of Biotechnology) was selected to treat the cells, with or without HG stimulation for $48 \mathrm{~h}$. The TXNIP inhibitor resveratrol (Res; $50 \mu \mathrm{mol} / \mathrm{l}$; Beyotime Institute of Biotechnology) was used to inhibit the
TXNIP/NLRP3 pathway. Cells were stimulated with Res for $2 \mathrm{~h}$ at $37^{\circ} \mathrm{C}$ in the dark and were then incubated under $\mathrm{HG}$ conditions for $48 \mathrm{~h}$. BMSCs were cultured to 4-7 generations for subsequent experiments.

Cell viability assay. Cell viability was determined using the Cell Counting Kit (CCK)-8 assay (Dojindo Molecular Technologies, Inc.). Firstly, cells were seeded in a 96-well plate at a density of $5 \times 10^{3}$ cells/well. The cells were grown to $\sim 90 \%$ confluence and were then co-cultured with various concentrations of FPS-ZM1 $(0,250,500$ or $750 \mathrm{nM})$ and HG $(25 \mathrm{mM})$ at $37^{\circ} \mathrm{C}$. Subsequently, the viability of the cultured cells was detected after 24,48 or $72 \mathrm{~h}$. The original medium was removed and $100 \mu \mathrm{l}$ serum-free DMEM containing $10 \mu \mathrm{l}$ CCK-8 solution was added to each well. Cells were subsequently incubated for $2 \mathrm{~h}$ at $37^{\circ} \mathrm{C}$ with $5 \% \mathrm{CO}_{2}$. Finally, a Varioskan Flash microplate reader (Thermo Fisher Scientific, Inc.) was used to detect the absorbance value at $450 \mathrm{~nm}$. The mean absorbance values of the control group obtained from the three different time-points were set as $100 \%$. Experiments were conducted three times.

$R N A$ isolation and reverse transcription-quantitative $P C R$ (RT-qPCR). The expression of specific genes (NLRP3, ASC, caspase-1, TXNIP and TRX) were quantitatively assessed by RT-qPCR. RNA was isolated from cultured BMSCs using TRIzol $^{\circledR}$ reagent (Invitrogen; Thermo Fisher Scientific, Inc.). Subsequently, RT of total RNA into cDNA was conducted using the Prime Script RT reagent kit (Takara Bio, Inc.), according to the manufacturer's protocol. SYBR FAST qPCR Master Mix (Kapa Biosystems; Roche Diagnostics) and a CFX-Connect 96 RT-qPCR system (Bio-Rad Laboratories, Inc.) were used to carry out the amplification of target genes. The PCR protocol was as follows: $95^{\circ} \mathrm{C}$ for $3 \mathrm{~min}$, followed by 40 cycles at $95^{\circ} \mathrm{C}$ for $5 \mathrm{sec}$ and $56^{\circ} \mathrm{C}$ for $10 \mathrm{sec}$, and a final extension step at $72^{\circ} \mathrm{C}$ for $25 \mathrm{sec}$. The mRNA levels of the specific genes were normalized to $\beta$-actin and expressed as a ratio to the internal reference. The primer sequences used for PCR are listed in Table I. The relative mRNA expression levels were calculated using the $2^{-\Delta \Delta \mathrm{Cq}}$ method and are presented as calculated values (31).

ELISA. Cell supernatants were obtained by centrifugation $(1,000 \mathrm{x} \mathrm{g}$; room temperature; $10 \mathrm{~min})$ after treatment at $48 \mathrm{~h}$ and stored at $-80^{\circ} \mathrm{C}$ for follow-up experiments. Activation of inflammatory factors was quantified using rat IL-1 $\beta$ (cat. no. RLB00), TNF- $\alpha$ (cat. no. RTA00) and IL-6 (cat. no. R6000B) ELISA kits (all R\&D Systems, Inc.), according to the manufacturer's protocols. Experiments were carried out three times.

Protein isolation and western blot analysis. The expression levels of specific proteins (NLRP3, ASC, caspase-1, TXNIP, TRX and RAGE) were detected by western blotting. Proteins were extracted from cultured BMSCs using RIPA buffer (Thermo Fisher Scientific, Inc.) at $4^{\circ} \mathrm{C}$ and total protein was boiled at $95^{\circ} \mathrm{C}$ for $10 \mathrm{~min}$ and centrifuged at room temperature at $12,000 \mathrm{x} \mathrm{g}$ for $10 \mathrm{~min}$. The bicinchoninic acid protein assay kit (Beijing Solarbio Science \& Technology, Co., Ltd.) was used for protein quantification. Protein samples $(20 \mu \mathrm{g})$ were 
Table I. Primer sequences used for reverse transcription-quantitative PCR.

\begin{tabular}{ll}
\hline Gene & \multicolumn{1}{c}{ Primer sequence $\left(5^{\prime}-3^{\prime}\right)$} \\
\hline NLRP3 & F-CATCTTAGTCCTGCCAA \\
& R-CAACAGACGCTACACCC \\
ASC & F-AGCATCCAGCAAACCA \\
& R-GGACCCCATAGACCTCA \\
Caspase-1 & F-TTGAAGAGCAGAAAGCA \\
& R-CAGTAGGAAACTCCGAAG \\
TXNIP & F-CAAGGTAAGTGTGCCG \\
& R-GATTCTGTGAAGGTGATGA \\
TRX & F-CCAACCTTTTGACCCTTT \\
& R-CCCTTCTTTCATTCCCTC \\
& F-TAGGAGCCAGGGCAGTA \\
& R-CGTTGACATCCGTAAAGAC
\end{tabular}

F, forward; R, reverse; NLRP3, nucleotide-binding oligomerization domain-like receptor protein 3; ASC, apoptosis-related speck-like protein containing CARD; TXNIP, thioredoxin-interacting protein; TRX, thioredoxin.

separated by $12 \%$ SDS-PAGE (Sigma-Aldrich; Merck KGaA) and were blotted onto polyvinylidene fluoride membranes (EMD Millipore). The membranes were then blocked in 5\% skim milk in PBS-0.05\% Tween-20 at room temperature for $1 \mathrm{~h}$. The membranes were then incubated with primary antibodies against RAGE (rabbit; 1:1,000; cat. no. PAB32996), NLRP3 (rabbit; 1:2,000; cat. no. PAB37930), ASC (rabbit; 1:1,000; cat. no. PAB30696), caspase-1 (rabbit; 1:1,000; cat.no. PAB36756), TXNIP (rabbit; 1:1,000; cat. no. PAB43948), TRX (rabbit; 1:1,000; cat. no. PAB32168) and $\beta$-actin (rabbit; 1:1,000; cat. no. PAB36265) (all Bioswamp) at $4^{\circ} \mathrm{C}$ overnight. $\beta$-actin was used as the loading control. Subsequently, membranes were incubated with horseradish peroxidase-conjugated goat anti-rabbit antibodies (1:20,000; cat. no. SAB43714; Bioswamp) for $1 \mathrm{~h}$ at room temperature. An enhanced chemiluminescence kit (Analytik Jena AG) was used to measure reactivity, and the target bands were detected, and protein expression was semi-quantified using TANON GIS 4.2 software (Tanon Science \& Technology Co., Ltd.). The detected protein expression levels were normalized to those of $\beta$-actin.

Statistical analysis. All data obtained from three experimental repeats are presented as the mean \pm standard deviation, and statistical analyses were conducted using SPSS 19.0 software (IBM Corp.). One-way analysis of variance was used for multiple group comparisons, and Tukey's post hoc test was used for intergroup comparisons. $\mathrm{P}<0.05$ was considered to indicate a statistically significant difference.

\section{Results}

FPS-ZM1 inhibits HG-induced cell viability. To verify suitable time-points and the optimal concentration of FPS-ZM1 for follow-up experiments, BMSCs were treated with FPS-ZM1 $(0,250,500$ and $750 \mathrm{nM})$ for 24,48 and $72 \mathrm{~h}$. Alterations in

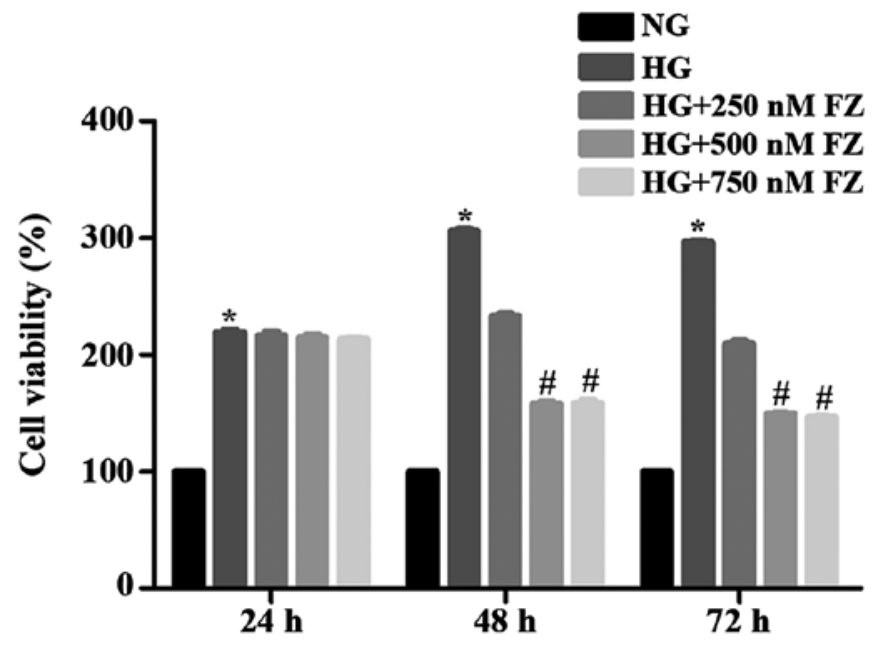

Figure 1. Effects of FZ (0, 250, 500 and $750 \mathrm{nM})$ on bone marrow mesenchymal stem cell viability at 24,48 and 72 h. Cell Counting Kit- 8 assay was performed after co-culturing cells with FPS-ZM1 and HG for $48 \mathrm{~h}$. Data are presented as the mean \pm standard deviation. ${ }^{*} \mathrm{P}<0.05$ vs. the NG group; ${ }^{\#} \mathrm{P}<0.05$ vs. the HG group. FZ, FPS-ZM1; HG, high glucose; NG, normal glucose.

BMSC viability are presented in Fig. 1. Compared with in the NG group, HG stimulation significantly enhanced the viability of BMSCs at all selected time points $(\mathrm{P}<0.05)$. Notably, no significant alterations in BMSC viability were detected following treatment with 250,500 or $750 \mathrm{nM}$ FPS-ZM1 for $24 \mathrm{~h}$. Conversely, treatment with 500 and 750 nM FPS-ZM1 for 48 or $72 \mathrm{~h}$ significantly alleviated $\mathrm{HG}$-induced cell viability compared with the HG group $(\mathrm{P}<0.05)$. No significant differences in viability were observed between cells treated with 500 or $750 \mathrm{nM}$ FPS-ZM1 for 48 and $72 \mathrm{~h}(\mathrm{P}>0.05)$. These results indicated that $\mathrm{HG}$ may promote the viability of BMSCs at $48 \mathrm{~h}$, but this effect may gradually decrease as the culture time increases.

FPS-ZM1 alleviates $H G$-induced inflammatory factor activation in BMSCs. ELISA kits were used to detect the levels of IL-6, TNF- $\alpha$ and IL-1 $\beta$ in BMSCs under different treatments. The impact of FPS-ZM1 on HG-induced intracellular inflammation was also assessed. As presented in Fig. 2, the concentrations of TNF- $\alpha$, IL- $1 \beta$ and IL- 6 were elevated under $\mathrm{HG}$ conditions compared with in the NG group $(\mathrm{P}<0.05)$. However, FPS-ZM1 significantly reduced the effects of $\mathrm{HG}$ on inflammatory marker levels $(\mathrm{P}<0.05)$. Similarly, the concentrations of TNF- $\alpha$, IL- $1 \beta$ and IL- 6 were decreased in BMSCs after pretreatment with Res $(\mathrm{P}<0.05)$.

FPS-ZM1 inhibits RAGE expression in HG-induced BMSCs. To explore the impact of FPS-ZM1 on RAGE expression in BMSCs under HG conditions, western blotting was used to assess the protein expression of RAGE. RAGE expression was increased in BMSCs under HG conditions, whereas treatment with FPS-ZM1 inhibited the increase in RAGE expression in HG-induced BMSCs $(\mathrm{P}<0.05)$ (Fig. 3).

FPS-ZM1 inhibits HG-induced TXNIP/NLRP3 inflammasome activation. The present study aimed to determine whether FPS-ZM1 inhibited inflammation through the 

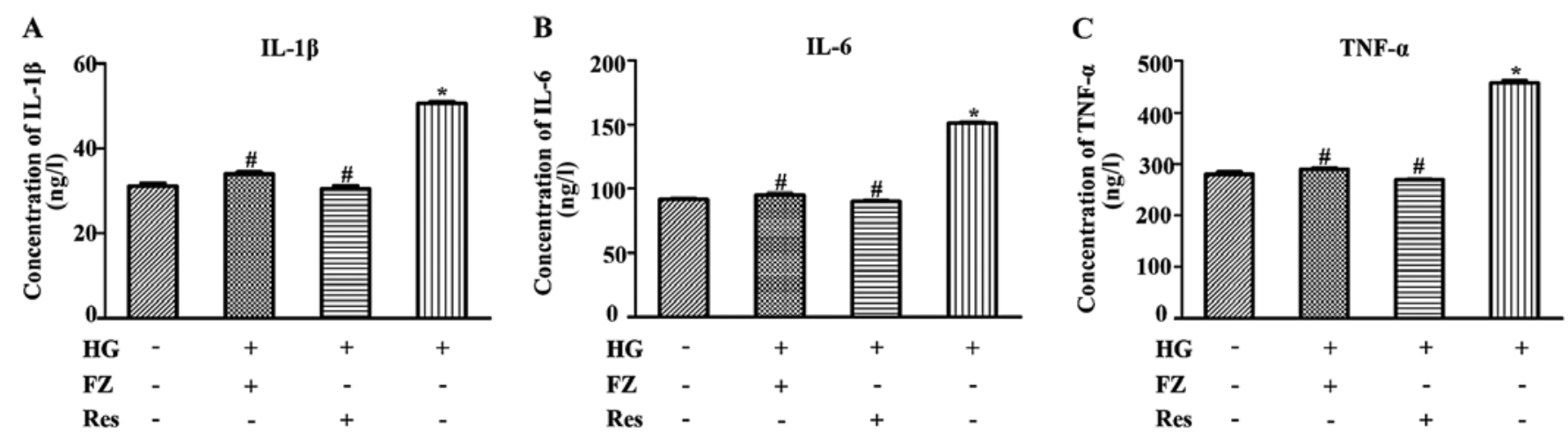

Figure 2. Effects of FZ treatment on the concentration of inflammatory factors in bone marrow mesenchymal stem cells. ELISA was performed after cells were pretreated with Res for $2 \mathrm{~h}$ or co-cultured with FZ and HG for $48 \mathrm{~h}$. Levels of (A) IL-1 $\beta$, (B) IL-6 and (C) TNF- $\alpha$ were analyzed. Data are presented as the mean \pm standard deviation. " $\mathrm{P}<0.05$ vs. the normal glucose group; ${ }^{\prime} \mathrm{P}<0.05$ vs. the HG group. FZ, FPS-ZM1; Res, resveratrol; HG, high glucose.

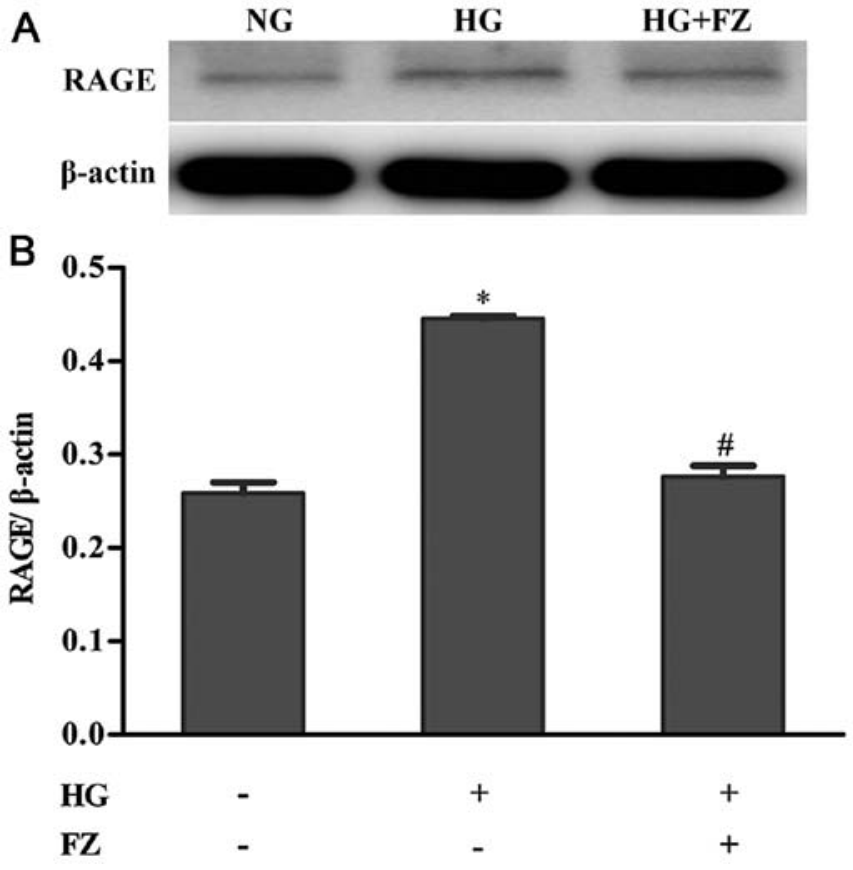

Figure 3. Effects of FZ on RAGE protein expression. Western blotting was performed after cells were co-cultured with FZ and HG for $48 \mathrm{~h}$. $\beta$-actin was used as the loading control. (A) RAGE protein was measured by western blotting. (B) The RAGE/ $\beta$-actin ratio was calculated to perform densitometric analysis of band intensity. Data are presented as the mean \pm standard deviation. ${ }^{*} \mathrm{P}<0.05$ vs. the NG group; ${ }^{\#} \mathrm{P}<0.05$ vs. the HG group. FZ, FPS-ZM1; RAGE, receptor for advanced glycation end products; NG, normal glucose; $\mathrm{HG}$, high glucose.

TXNIP/TRX/NLRP3 inflammasome signaling pathway. As presented in Fig. 4, the mRNA expression of TXNIP, NLRP3, ASC and caspase-1 were increased, whereas TRX expression levels were decreased in $\mathrm{HG}$-induced cells $(\mathrm{P}<0.05)$. Treatment with FPS-ZM1 or pretreatment with Res reduced the expression of TXNIP, caspase-1, NLRP3 and ASC, and enhanced TRX expression $(\mathrm{P}<0.05)$. Similar results were determined by western blotting; TXNIP, NLRP3, ASC and caspase-1 levels were increased, whereas TRX expression was decreased in $\mathrm{HG}$-induced BMSCs $(\mathrm{P}<0.05)$. However, these effects were significantly reversed by FPS-ZM1 or Res $(\mathrm{P}<0.05)$ (Fig. 5). These results indicated that FPS-ZM1 may inhibit TXNIP/NLRP3 activation under a HG environment.

\section{Discussion}

AGEs have been reported to aggregate within the oral periodontal tissues of patients with diabetes, contributing to the inflammatory process in the surrounding soft and hard tissues $(32,33)$. Inflammation may be enhanced when AGEs bind to RAGE, thus resulting in upregulation of various proinflammatory factors (34). IL-1 $\beta$ is considered the most important cytokine, which serves a crucial role in the inflammatory process (35). IL-6 is a proinflammatory cytokine that is closely related to periodontitis and rheumatoid arthritis (36). TNF- $\alpha$ promotes T cells by secreting various activated cytokines, which are closely associated with autoimmune diseases, inflammation and diabetes (37).

RAGE is highly expressed in numerous cell types in periodontal tissues under HG stimulation, including BMSCs $(18,38)$. It is well known that BMSCs are characterized by multidirectional differentiation and low immunity, and the potential immune-regulating effect of BMSCs has garnered increasing attention. BMSCs are functional and pivotal cells that repair damaged tissues and organs, including bone tissue, cartilage and joint injury (39). However, the differentiation of BMSCs into osteoblasts, lipoblasts or chondroblasts can be inhibited by inflammation, thus affecting the tissue repair process (40). BMSC inflammation has been reported to respond to $\mathrm{HG}$; a previous study revealed that BMSCs isolated from diabetic rats exhibited stronger expression of $N F-\kappa B$ and IL-18 compared with normal rats (41). The present study assessed the inflammatory response of BMSCs induced by $\mathrm{HG}$ in vitro. Subsequently, the effects of FPS-ZM1 on regulating HG-induced inflammation and the underlying potential mechanism were determined.

In the present study, the effects of HG were detected on RAGE expression in BMSCs; the results indicated that $\mathrm{HG}$ exposure stimulated the increased expression of RAGE in BMSCs. However, FPS-ZM1, a RAGE-specific inhibitor, reversed the enhanced expression of RAGE induced by HG. In addition, the effects of various concentrations of FPS-ZM1 $(0,250,500$ and $750 \mathrm{nM})$ for different durations on BMSCs were examined. The viability of BMSCs was enhanced under HG conditions. Conversely, FPS-ZM1 alleviated HG-induced viability. The present study identified significant differences between the NG group and the HG-treated groups regardless 

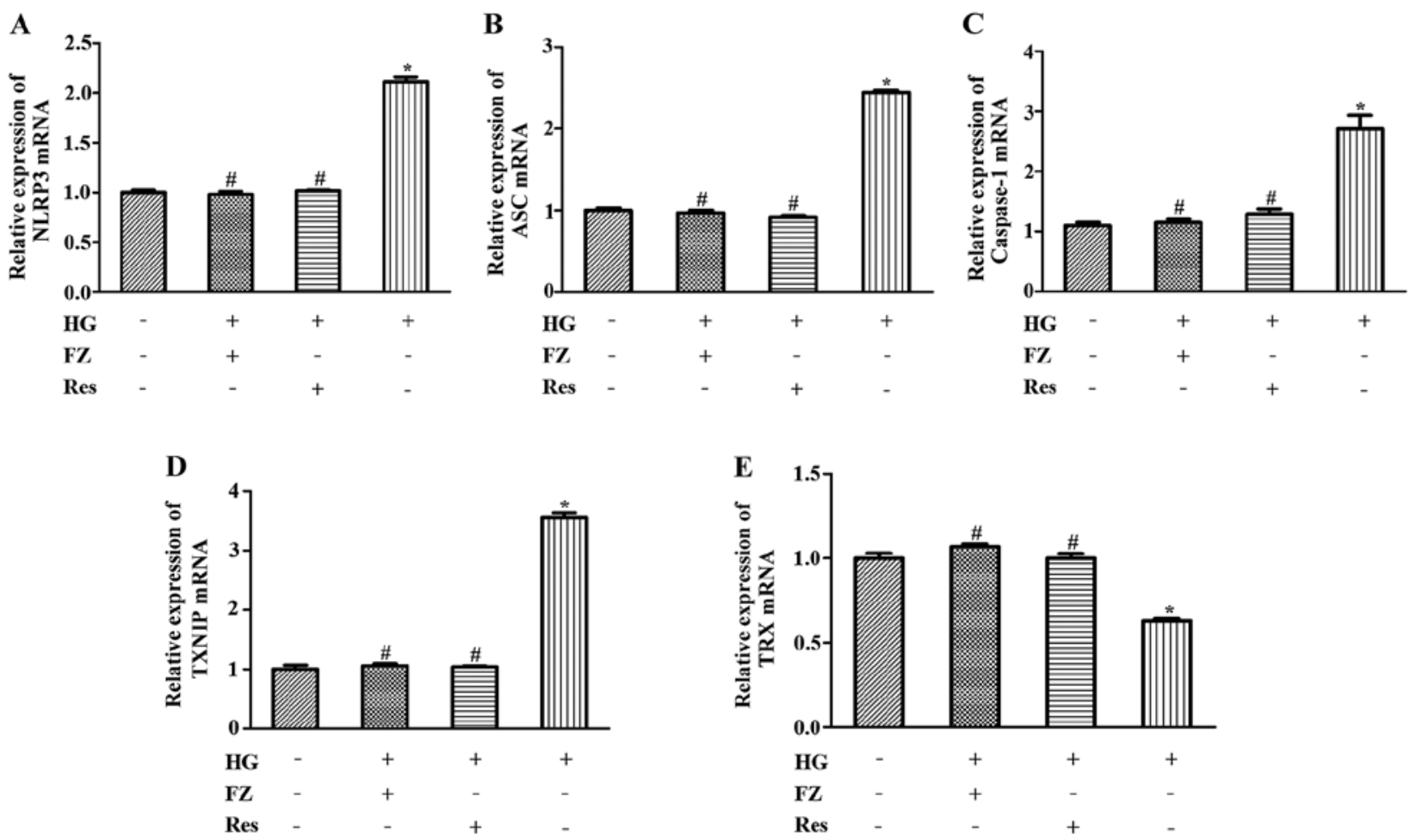

Figure 4. Effects of FZ on the mRNA expression levels of ASC, TXNIP, NLRP3, caspase-1 and TRX. Reverse transcription-quantitative PCR was performed after cells were pretreated with Res for $2 \mathrm{~h}$ or co-cultured with FZ and HG for $48 \mathrm{~h}$. (A) NLRP3, (B) ASC, (C) caspase-1, (D) TXNIP and (E) TRX mRNA expression. Data are presented as the mean \pm standard deviation. ${ }^{*} \mathrm{P}<0.05$ vs. the normal glucose group; ${ }^{\mathrm{P}} \mathrm{P}<0.05$ vs. the HG group. FZ, FPS-ZM1; ASC, apoptosis-related speck-like protein containing CARD; TXNIP, thioredoxin-interacting protein; NLRP3, nucleotide-binding oligomerization domain-like receptor protein 3; TRX, thioredoxin; HG, high glucose; Res, resveratrol.

A

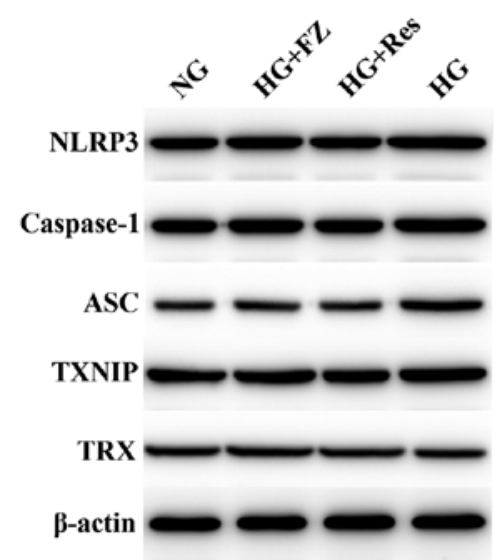

B

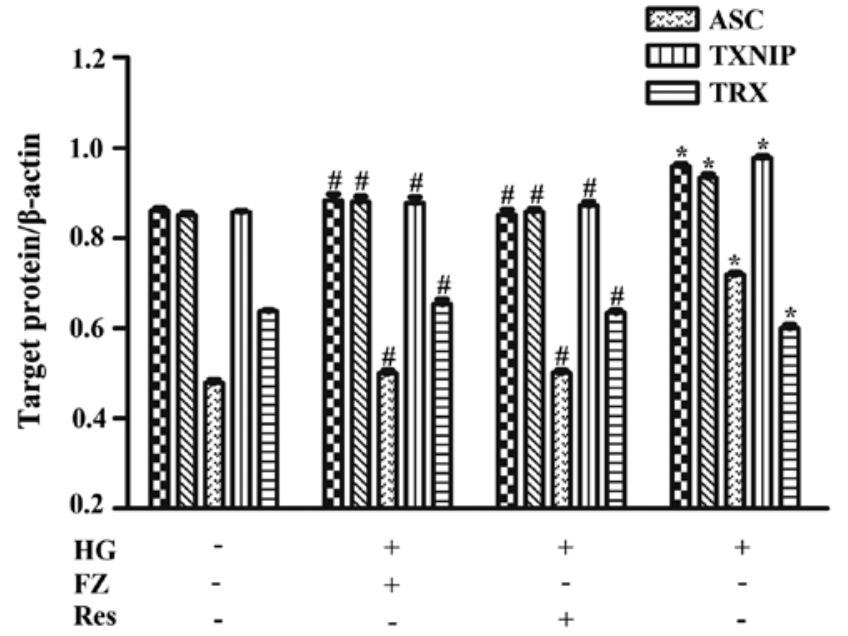

Figure 5. Effect of FZ on target protein expression levels, including ASC, TXNIP, NLRP3, caspase-1 and TRX. Western blot analysis was performed after cells were pretreated with Res for $2 \mathrm{~h}$ or co-cultured with FPS-ZM1 and HG for $48 \mathrm{~h}$. $\beta$-actin was used as a loading control. (A) Protein expression levels of NLRP3, caspase-1, ASC, TXNIP and TRX were measured by western blotting. (B) NLRP3/ $\beta$-actin, caspase- $1 / \beta$-actin, ASC $/ \beta$-actin, TXNIP/ $\beta$-actin and TRX/ $\beta$-actin ratios were calculated to perform densitometric analysis of band intensity. Data are presented as the mean \pm standard deviation. ${ }^{*} \mathrm{P}<0.05$ vs. the NG group. ${ }^{\text {\#P}} \mathrm{P}<0.05$ vs. the HG group. FZ, FPS-ZM1; ASC, apoptosis-related speck-like protein containing CARD; TXNIP, thioredoxin-interacting protein; NLRP3, nucleotide-binding oligomerization domain-like receptor protein 3; TRX, thioredoxin; NG, normal glucose; HG, high glucose; Res, resveratrol.

of treatment duration. Cell viability of the HG group was significantly increased compared with NG group, and peaked at $48 \mathrm{~h}$; however, the cell viability began to decrease from $72 \mathrm{~h}$, which might be due to time-dependent HG aggravation of oxidative stress in BMSCs $(42,43)$. It has been reported that AGE expression can significantly inhibit the viability of cells, such as fibroblasts, in a time-dependent manner (44). AGEs may destroy stability of the internal environment by enhancing intracellular oxidative stress, thus inhibiting cell viability (45). 
The present study assessed the effects of FPS-ZM1 on HG-stimulated inflammation in BMSCs. It was previously observed that $\mathrm{HG}$ conditions promoted inflammatory factors and inflammatory cytokines, such as TNF- $\alpha$, IL-1 $\beta$ and IL-6, in periodontal tissues $(46,47)$. The present study on BMSCs presented similar findings; TNF- $\alpha$, IL-1 $\beta$ and IL- 6 expression was enhanced after HG stimulation, whereas these effects were inhibited by FPS-ZM1. FPS-ZM1 exerted a protective effect against $\mathrm{HG}$ conditions in vitro; however, the mechanism underlying the anti-inflammatory effects of FPS-ZM1 has yet to be determined.

The present study indicated that the TXNIP/NLRP3 pathway may serve a role as the intracellular transduction pathway underlying the regulatory effects of FPS-ZM1 on HG-induced inflammation. The NLRP3 inflammasome is a protein complex that regulates inflammation and cell death (48). Previous studies have reported that NLRP3 can be overexpressed in epithelial cells or fibroblasts by glucose, oxidative stress and other types of stimulation, and it has been suggested to affect diabetes-related periodontal diseases $(49,50)$. TXNIP has been recognized as an early mediator associated with diabetic inflammation (51). TXNIP binds to TRX and inhibits its activity; therefore, TXNIP is also called the TRX-binding protein (52). Hyperglycemia may upregulate TXNIP by directly activating the NLRP 3 inflammasome, followed by an enhancement of inflammation-activated factors (53). In the present study, FPS-ZM1 downregulated HG-induced expression of TXNIP and NLRP3. Furthermore, the downregulation of TRX expression stimulated by HG was reversed by FPS-ZM1. These findings confirmed the role of the TXNIP/NLRP3 inflammasome signaling pathway in the regulatory effects of FPS-ZM1 on HG-induced BMSC. This pathway was also inactivated by Res.

A previous study reported that Res significantly inhibited the activity of the TXNIP/NLRP3 inflammasome pathway to achieve the pharmacological effect of inhibiting inflammation (54). In the present study, pretreatment with Res disrupted the activity of the TXNIP/NLRP3 inflammasome pathway. Furthermore, Res downregulated HG-induced expression of TNF- $\alpha$, IL-1 $\beta$ and IL-6. These findings further support the hypothesis that the TXNIP/NLRP3 inflammasome signaling pathway is involved in HG-stimulated inflammation. Therefore, inhibition of the TXNIP/NLRP3 inflammasome signaling pathway may be considered one of the mechanisms underlying the anti-inflammatory effects of FPS-ZM1.

The present study also revealed that although the mRNA and protein expression levels of inflammation-associated molecules, such as NLRP3, caspase-1, ASC and TXNIP, were higher in the HG group than in the NG group, there was a marked difference between mRNA and protein expression levels. There exists a linear correlation between mRNA and protein expression, and the change ratio should be the same; however, the results of other experimental studies have demonstrated that the linear correlation is not very high $(55,56)$. This phenomenon has been reported by numerous studies and may be explained as follows: Gene expression is regulated in numerous ways, not just by transcriptional regulation (e.g., histone modification, DNA methylation and transcription factor regulation), but also by post-transcriptional alterations, such as microRNA targeting,
RNA-binding proteins or RNA modification (57-59). In addition, various factors, including mRNA degradation, protein degradation and protein folding may lead to differences between mRNA and protein expression levels $(60,61)$. How the reasons for the differences observed between the mRNA and protein expression remain unknown and should be further investigated in the future.

In conclusion, the regulatory effects of FPS-ZM1 on HG-induced inflammation and NLRP3 accumulation in BMSCs were investigated. HG stimulated RAGE expression in BMSCs and mediated a series of inflammatory responses, whereas treatment with FPS-ZM1, a RAGE-specific inhibitor, protected BMSCs by exerting anti-inflammatory effects, specifically reducing HG-induced cell viability, inflammatory factor production and TXNIP/NLRP3 inflammasome signaling pathway activation. In addition, inactivation of the TXNIP/NLRP3 inflammasome by Res exhibited similar effects as FPS-ZM1, which indicated that the molecular mechanism underlying the anti-inflammatory effect of FPS-ZM1 may be associated with the TXNIP/NLRP3 inflammasome signaling pathway. Based on the anti-inflammatory effects, the results of the present study provided a novel therapeutic approach and target drug therapy for patients with diabetes with accelerated occurrence and development of periodontal diseases. Nonetheless, these studies were limited to in vitro experiments on BMSCs. Further studies regarding the role of FPS-ZM1 on peri-implantitis or periodontal tissues in animal models are required, as the human microenvironment is more complicated, and an assessment of the response in vivo is necessary.

\section{Acknowledgements}

Not applicable.

\section{Funding}

The present study was supported by the Luzhou-school Union (grant no. 2016LZXNYD-J20).

\section{Availability of data and materials}

The datasets used and/or analyzed during the current study are available from the corresponding author on reasonable request.

\section{Authors' contributions}

MJ and LG mainly contributed to experimental conception and design. MJ, XW and PW performed the experiments and collected the data. MJ, WP and BZ conducted the statistical analysis. MJ wrote the original draft. XW, PW and LG revised the completed draft. All authors read and approved the final manuscript.

\section{Ethics approval and consent to participate}

Not applicable.

\section{Patient consent for publication}

Not applicable. 


\section{Competing interests}

The authors declare that they have no competing interests.

\section{References}

1. Shi Y and Hu FB: The global implications of diabetes and cancer. Lancet 383: 1947-1948, 2014.

2. Yamamoto Y and Yamamoto H: RAGE-Mediated Inflammation, type 2 diabetes, and diabetic vascular complication. Front Endocrinol (Lausanne) 4: 105, 2013.

3. Rübsam A, Parikh S and Fort PE: Role of Inflammation in diabetic retinopathy. Int J Mol Sci 19: 942, 2018.

4. Yuan Y, Sun H and Sun Z: Advanced glycation end products (AGEs) increase renal lipid accumulation: A pathogenic factor of diabetic nephropathy (DN). Lipids Health Dis 16: 126, 2017.

5. Shi L, Yu X, Yang H and Wu X: Advanced glycation end products induce human corneal epithelial cells apoptosis through generation of reactive oxygen species and activation of JNK and p38 MAPK pathways. PLoS One 8: e66781, 2013.

6. Meloche J, Paulin R, Roy M, Agharazii M and Bonnet S: Activation of RAGE/STAT3 axis by advanced glycation endproducts in vascular remodeling diseases. Circulation 122 (Suppl 21): S15969, 2010.

7. Sanajou D, Haghjo AG, Argani H and Aslani S: AGE-RAGE axis blockade in diabetic nephropathy: Current status and future directions. Eur J Pharmacol 833: 158-164, 2018.

8. Sanajou D, Ghorbani Haghjo A, Argani H, Roshangar L, Rashtchizadeh N, Ahmad SNS, Ashrafi-Jigheh Z and Bahrambeigi S: Reduction of renal tubular injury with a RAGE inhibitor FPS-ZM1, valsartan and their combination in streptozotocin-induced diabetes in the rat. Eur J Pharmacol 842: 40-48, 2019.

9. Zhan D, Guo L and Zheng L: Inhibition of the receptor for advanced glycation promotes proliferation and repair of human periodontal ligament fibroblasts in response to high glucose via the NF-kappa B signaling pathway. Arch Oral Biol 87: 86-93, 2018.

10. Huang J, Xiong T, Zhang Z, Tan Y and Guo L: Inhibition of the receptor for advanced glycation inhibits lipopolysaccharide-mediated High mobility group protein B1 and Interleukin-6 synthesis in human gingival fibroblasts through the NF- $\mathrm{KB}$ signaling pathway. Arch Oral Biol 105: 81-87, 2019.

11. Graves DT, Ding Z and Yang Y: The impact of diabetes on periodontal diseases. Periodontol 82: 214-224, 2020.

12. Almajwal AM, Alam I, Abulmeaty M, Razak S, Pawelec G and Alam W: Intake of dietary advanced glycation end products influences inflammatory markers, Immune phenotypes, and antiradical capacity of healthy elderly in a little-studied population. Food Sci Nutr 8: 1046-1057, 2020

13. Yao D, Wang S, Wang M and Lu W: Renoprotection of dapagliflozin in human renal proximal tubular cells via the inhibition of the high mobility group box 1receptor for advanced glycation end productsnuclear factor- $\kappa \mathrm{B}$ signaling pathway. Mol Med Rep 18: 3625-3630, 2018.

14. Chang PC, Chien LY, Yeo JF, Wang YP, Chung MC, Chong LY, Kuo MY, Chen CH, Chiang HC, Ng BN, et al: Progression of periodontal destruction and the roles of advanced glycation end products in experimental diabetes. J Periodontol 84: 379-388, 2013.

15. Bartold PM and Van Dyke TE: Periodontitis: A host-mediated disruption of microbial homeostasis. Unlearning learned concepts. Periodontol 62: 203-217, 2013

16. Al-Sowygh ZH, Ghani SMA, Sergis K, Vohra F and Akram Z: Peri-implant conditions and levels of advanced glycation end products among patients with different glycemic control. Clin Implant Dent Relat Res 20: 345-351, 2018.

17. Dey D, Jingar P, Agrawal S, Shrivastava V, Bhattacharya A, Manhas J, Garg B, Ansari MT, Mridha AR, Sreenivas V, et al: Symphytum officinale augments osteogenesis in human bone marrow-derived mesenchymal stem cells in vitro as they differentiate into osteoblasts. J Ethnopharmacol 248: 112329, 2020.

18. Dhanasekaran M, Indumathi S, Rajkumar JS and Sudarsanam D: Effect of high glucose on extensive culturing of mesenchymal stem cells derived from subcutaneous fat, omentum fat and bone marrow. Cell Biochem Funct 31: 20-29, 2013.
19. Nonaka K, Kajiura Y, Bando M, Sakamoto E, Inagaki Y, Lew JH, Naruishi K, Ikuta T, Yoshida K, Kobayashi T, et al: Advanced glycation end-products increase IL-6 and ICAM-1 expression via RAGE, MAPK and NF- $\kappa$ B pathways in human gingival fibroblasts. J Periodontal Res 53: 334-344, 2018.

20. Thielen L and Shalev A: Diabetes pathogenic mechanisms and potential new therapies based upon a novel target called TXNIP. Curr Opin Endocrinol Diabetes Obes 25: 75-80, 2018.

21. Gouravani M, Khalili N, Razi S, Keshavarz-Fathi M, Khalili N and Rezaei N: The NLRP3 inflammasome: A therapeutic target for inflammation-associated cancers. Expert Rev Clin Immunol 16: 175-187, 2020.

22. Feng H, Gu J, Gou F, Huang W, Gao C, Chen G, Long Y, Zhou X, Yang M, Liu S, et al: High Glucose and lipopolysaccharide prime NLRP3 inflammasome via ROS/TXNIP pathway in mesangial cells. J Diabetes Res 2016: 1-11, 2016.

23. An N, Gao Y, Si Z, Zhang H, Wang L, Tian C, Yuan M, Yang X, Li X, Shang H, et al: Regulatory mechanisms of the NLRP3 inflammasome, a novel immune-inflammatory marker in cardiovascular diseases. Front Immunol 10: 1592, 2019.

24. Matsui T, Nakamura N, Ojima A, Nishino Y and Yamagishi SI: Sulforaphane reduces advanced glycation end products (AGEs)-induced inflammation in endothelial cells and rat aorta. Nutr Metab Cardiovasc Dis 26: 797-807, 2016.

25. Saresella M, La Rosa F, Piancone F, Zoppis M, Marventano I, Calabrese E, Rainone V, Nemni R, Mancuso R and Clerici M: The NLRP3 and NLRP1 inflammasomes are activated in Alzheimer's disease. Mol Neurodegener 11: 23, 2016.

26. Zhao LR, Xing RL, Wang PM, Zhang NS, Yin SJ, Li XC and Zhang L: NLRP ${ }_{1}$ and $\mathrm{NLRP}_{3}$ inflammasomes mediate LPS/ATP-induced pyroptosis in knee osteoarthritis. Mol Med Rep 17: 5463-5469, 2018.

27. Wen H, Gris D, Lei Y, Jha S, Zhang L, Huang MT, Brickey WJ and Ting JP: Fatty acid-induced NLRP3-ASC inflammasome activation interferes with insulin signaling. Nat Immunol 12: 408-415, 2011.

28. Bharti V, Tan H, Zhou $\mathrm{H}$ and Wang JF: Txnip mediates glucocorticoid-activated NLRP3 inflammatory signaling in mouse microglia. Neurochem Int 131: 104564, 2019.

29. Omar DF, Kamal MM, El-Hefnawy MH and El-Mesallamy HO: Serum Vitamin D and its upregulated protein, thioredoxin interacting protein, are associated with beta-cell dysfunction in adult patients with type 1 and type 2 diabetes. Can J Diabetes 42 : 588-594, 2018.

30. Gateva AT, Assyov YS, Velikova T and Kamenov ZA: Higher levels of thioredoxin interacting protein (TXNIP) in patients with prediabetes compared to obese normoglycemic subjects. Diabetes Metabolic Syndrome 13: 734-737, 2019.

31. Livak KJ and Schmittgen TD: Analysis of relative gene expression data using real-time quantitative PCR and the 2(-Delta Delta C(T)) method. Methods 25: 402-408, 2001

32. Chiu HC, Fu MM, Yang TS, Fu E, Chiang CY, Tu HP, Chin YT, Lin FG and Shih KC: Effect of high glucose, Porphyromonas gingivalis lipopolysaccharide and advanced glycation endproducts on production of interleukin-6/-8 by gingival fibroblasts. J Periodontal Res 52: 268-276, 2017

33. Lalla E, Lamster IB, Stern DM and Schmidt AM: Receptor for advanced glycation end products, inflammation, and accelerated periodontal disease in diabetes: Mechanisms and insights into therapeutic modalities. Ann Periodontol 6: 113-118, 2001.

34. Daniele G, Guardado Mendoza R, Winnier D, Fiorentino TV, Pengou Z, Cornell J, Andreozzi F, Jenkinson C, Cersosimo E, Federici M, et al: The inflammatory status score including IL- 6 , TNF- $\alpha$, osteopontin, fractalkine, MCP-1 and adiponectin underlies whole-body insulin resistance and hyperglycemia in type 2 diabetes mellitus. Acta Diabetol 51: 123-131, 2014.

35. Han Y, Xu X, Tang C, Gao P, Chen X, Xiong X, Yang M, Yang S, Zhu X, Yuan S, et al: Reactive oxygen species promote tubular injury in diabetic nephropathy: The role of the mitochondrial ros-txnip-nlrp3 biological axis. Redox Biol 16: 32-46, 2018.

36. Taylor JJ, Preshaw PM and Lalla E: A review of the evidence for pathogenic mechanisms that may link periodontitis and diabetes. J Clin Periodontol 40 (4 Suppl): S113-S134, 2013.

37. Akdis M, Aab A, Altunbulakli C, Azkur K, Costa RA, Crameri R, Duan S, Eiwegger T, Eljaszewicz A, Ferstl R, et al: Interleukins (from IL-1 to IL-38), interferons, transforming growth factor $\beta$, and TNF- $\alpha$ : Receptors, functions, and roles in diseases. J Allergy Clin Immunol 138: 984-1010, 2016. 
38. Zheng DH, Han ZQ, Wang XX, Ma D and Zhang J: Erythropoietin attenuates high glucose-induced oxidative stress and inhibition of osteogenic differentiation in periodontal ligament stem cell (PDLSCs). Chem Biol Interact 305: 40-47, 2019.

39. Kang K, Chuai JB, Xie BD, Li JZ, Qu H, Wu H, Fang SH, Cui JJ, Xiu LL, Han JC, et al: Mesenchymal stromal cells from patients with cyanotic congenital heart disease are optimal candidate for cardiac tissue engineering. Biomaterials 230: 119574, 2020.

40. Dong H, Wang Z, Chen Y and Li Y: Protective effects of bone Marrow-derived mesenchymal stem cells on insulin secretion and inflammation in the treatment of severe acute pancreatitis in rats. Transplant Proc 52: 333-344, 2020.

41. Li H, Zhou HL, Guo F, Wang Q and Li W: The effects of 25-hydroxyvitamin $\mathrm{D}_{3}$ on the activation of NLRP3 inflammasome and inflammatory response of bone marrow mesenchymal stem cells from the diabetic rats. J Pract Stomatol 34: 11-15, 2018.

42. Liao F, Liu Y, Liu HH, Hu J, Zhao S and Yang SM: Effect of Angelica sinensis polysaccharide on the osteogenic differentiation of bone marrow mesenchymal stem cells of rats with high glucose levels. Hua Xi Kou Qiang Yi Xue Za Zhi 37: 193-199, 2019 (In Chinese).

43. Li H, Wang D, Chen Y and Yang M: $\beta$-Caryophyllene inhibits high glucose-induced oxidative stress, inflammation and extracellular matrix accumulation in mesangial cells. Int Immunopharmacol 84: 106556, 2020.

44. Soydas T, Yaprak Sarac E, Cinar S, Dogan S, Solakoglu S, Tuncdemir M and Kanigur Sultuybek G: The protective effects of metformin in an in vitro model of aging 3T3 fibroblast under the high glucose conditions. J Physiol Biochem 74: 273-281, 2018.

45. Wu J, Lu K, Zhu M, Xie X, Ding Y, Shao X, Chen Y, Liu J, Xu M, $\mathrm{Xu}$ Y, et al: $\mathrm{miR}-485$ suppresses inflammation and proliferation of mesangial cells in an in vitro model of diabetic nephropathy by targeting NOX5. Biochem Biophys Res Commun 521: 984-990, 2020 .

46. Wang Z, Maruyama K, Sakisaka Y, Suzuki S, Tada H, Suto M, Saito M, Yamada $S$ and Nemoto E: Cyclic stretch force induces periodontal ligament cells to secrete exosomes that suppress IL- $1 \beta$ production through the inhibition of the NF- $\kappa \mathrm{B}$ signaling pathway in macrophages. Front Immunol 10: 1310, 2019.

47. Yang L, Wu L, Zhang X, Hu Y,Fan Y and Ma J: 1,25(OH)2D3/VDR attenuates high glucoseinduced epithelialmesenchymal transition in human peritoneal mesothelial cells via the TGF $\beta / S m a d 3$ pathway. Mol Med Rep 15: 2273-2279, 2017.

48. Dong F, Dong S, Liang Y, Wang K, Qin Y and Zhao X: miR-20b inhibits the senescence of human umbilical vein endothelial cells through regulating the $\mathrm{Wnt} / \beta$-catenin pathway via the TXNIP/NLRP3 axis. Int J Mol Med 45: 847-857, 2020.

49. Lu WL, Song DZ, Yue JL, Wang TT, Zhou XD, Zhang P, Zhang L and Huang DM: NLRP3 inflammasome may regulate inflammatory response of human periodontal ligament fibroblasts in an apoptosis-associated speck-like protein containing a CARD (ASC)-dependent manner. Int Endod J 50: 967-975, 2017.
50. Bui FQ, Johnson L, Roberts J, Hung SC, Lee J, Atanasova KR, Huang PR, Yilmaz Ö and Ojcius DM: Fusobacterium nucleatum infection of gingival epithelial cells leads to NLRP3 inflammasome-dependent secretion of IL- $1 \beta$ and the danger signals ASC and HMGB1. Cell Microbiol 18: 970-981, 2016.

51. Szpigel A, Hainault I, Carlier A, Venteclef N, Batto AF, Hajduch E, Bernard C, Ktorza A, Gautier JF, Ferré P, et al: Lipid environment induces ER stress, TXNIP expression and inflammation in immune cells of individuals with type 2 diabetes. Diabetologia 61: 399-412, 2018.

52. Lu L, Lu Q, Chen W, Li J, Li C and Zheng Z: Vitamin $\mathrm{D}_{3}$ Protects against diabetic retinopathy by inhibiting high-glucose-induced activation of the ROS/TXNIP/NLRP3 inflammasome pathway. J Diabetes Res 2018: 8193523, 2018.

53. Yi X, Zhang L, Lu W, Tan X, Yue J, Wang P, Xu W, Ye L and Huang D: The effect of NLRP inflammasome on the regulation of AGEs-induced inflammatory response in human periodontal ligament cells. J Periodontal Res 54: 681-689, 2019.

54. Bedarida T, Baron S, Vibert F, Ayer A, Henrion D, Thioulouse E, Marchiol C, Beaudeux JL, Cottart CH and Nivet-Antoine V: Resveratrol decreases TXNIP mRNA and protein nuclear expressions with an arterial function improvement in old mice. J Gerontol A Biol Sci Med Sci 71: 720-729, 2016.

55. Choe D, Palsson B and Cho BK: STATR: A simple analysis pipeline of Ribo-Seq in bacteria. J Microbiol 58: 217-226, 2020.

56. Jeong Y, Kim JN, Kim MW, Bucca G, Cho S, Yoon YJ, Kim BG, Roe JH, Kim SC, Smith CP, et al: The dynamic transcriptional and translational landscape of the model antibiotic producer Streptomyces coelicolor. Nat Commun 7: 11605, 2016.

57. Liu Y, Beyer A and Aebersold R: On the dependency of cellular protein levels on mRNA abundance. Cell 165: 535-550, 2016.

58. Koussounadis A, Langdon SP, Um IH, Harrison DJ and Smith VA: Relationship between differentially expressed mRNA and mRNA-protein correlations in a xenograft model system. Sci Rep 5: 10775, 2015.

59. Schwanhäusser B, Busse D, Li N, Dittmar G, Schuchhardt J, Wolf J, Chen W and Selbach M: Global quantification of mammalian gene expression control. Nature 473: 337-342, 2011.

60. Surkova S, Sokolkova A, Kozlov K, Nuzhdin SV and Samsonova M: Quantitative analysis reveals genotype- and domain-specific differences between mRNA and protein expression of segmentation genes in Drosophila. Dev Biol 448: 48-58, 2019.

61. Wu X, Zhao W, Cui Q and Zhou Y: Computational screening of potential regulators for mRNA-protein expression level discrepancy. Biochem Biophys Res Commun 523: 196-201, 2020.

This work is licensed under a Creative Commons Attribution-NonCommercial-NoDerivatives 4.0 International (CC BY-NC-ND 4.0) License. 